\title{
Corrigendum: Formation of nanodiamonds at near-ambient conditions via microplasma dissociation of ethanol vapour
}

\author{
Ajay Kumar, Pin Ann Lin, Albert Xue, Boyi Hao, Yoke Khin Yap \& R. Mohan Sankaran
}

Nature Communications 4:2618 doi: 10.1038/ncomms3618 (2013); Published 21 Oct 2013; Updated 3 Jul 2014

During the final stages of manuscript preparation, the fast Fourier transform (FFT) filtered images in the insets of Fig. 5a,b were inadvertently switched and labelled with the wrong crystallographic indices. Our analysis and conclusions remain unchanged by this error. The correct version of Fig. 5 appears below.
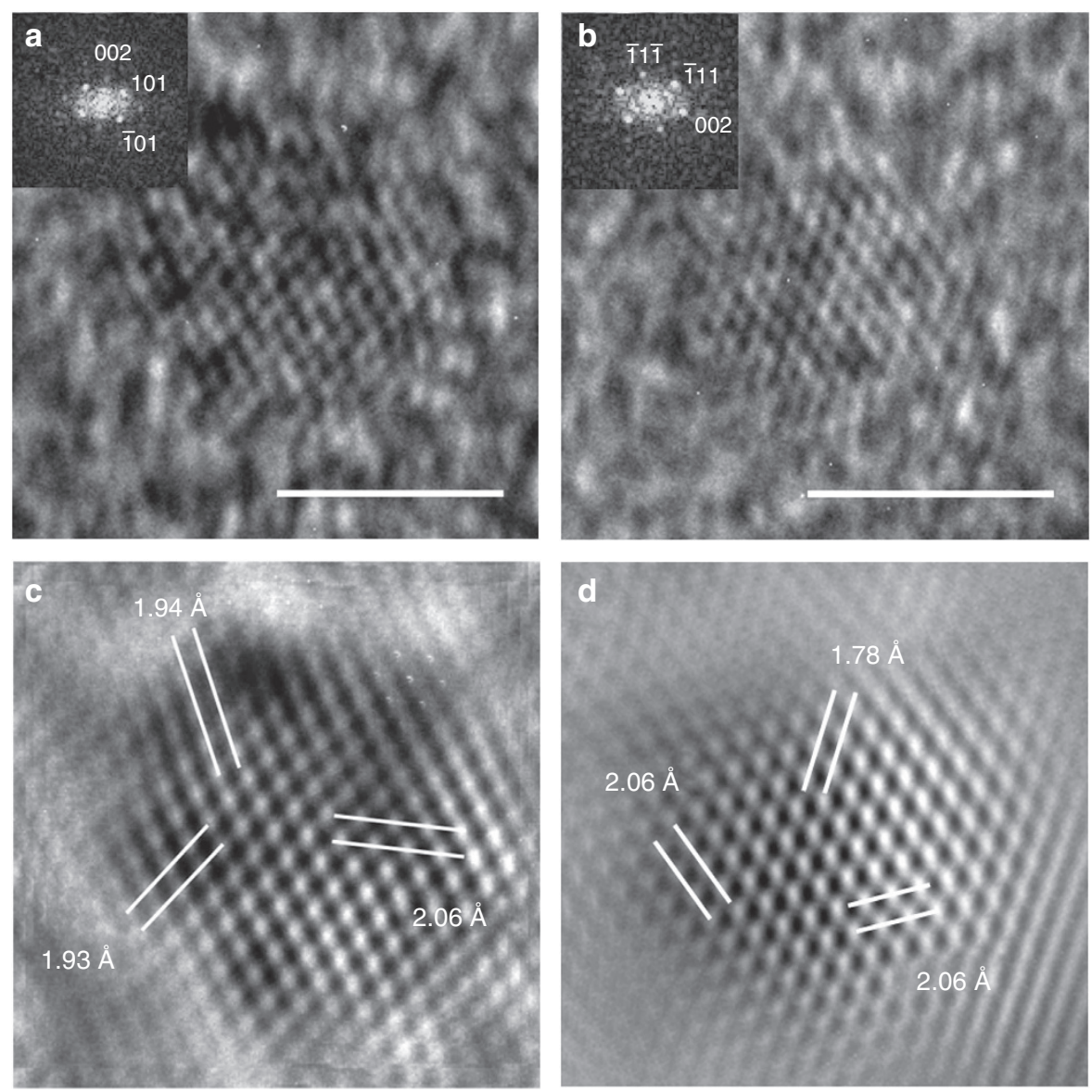